\title{
O impacto dos cenários de prática propostos pelo Pró-Saúde na formação em odontologia
}

\author{
Carlos Rafael do Carmo Rodrigues*, Marcos Alex Mendes da Silva** \\ * Graduando em Odontologia pela Universidade Severino Sombra, \\ Vassouras, RJ, Brasil \\ ** Docente do Curso de Odontologia da Universidade Severino \\ Sombra, Vassouras, RJ, Brasil
}

\section{RESUMO}

O Ministério da Saúde, por meio da Secretaria de Gestão e Educação no trabalho, elaborou o Programa Nacional de Reorientação da Formação Profissional em Saúde como um incentivo aos cursos da área da saúde para promoverem uma mudança de cenários na prática do ensino, introduzindo assim os alunos no Sistema Único de Saúde, a fim de mostra-lhes as reais demandas da população e prepará-los para o mercado de trabalho. O objetivo deste estudo consistiu em realizar uma análise nos cursos de odontologia do Brasil, contemplados com o Pró-Saúde, entrevistando seus coordenadores, sobre o impacto das ações do programa no ensino e sobre o quanto eles estão informados em relação as ações do Pró-Saúde. Foi aplicado um questionário estruturado de forma virtual aos 45 coordenadores de graduação em odontologia no Brasil após aprovação do Comitê de Ética e Pesquisa da Universidade Severino Sombra, sob o parecer de número 008/2012-1. Os resultados preliminares apontaram que $25 \%$ perceberam que o programa influenciou tanto na estrutura física, quanto na matriz curricular, onde, em $67 \%$ das universidades foi acrescentado uma carga horária extra destinada a estágios nas Unidades Básicas de Saúde. Apesar dos coordenadores afirmarem que em $50 \%$ das universidades, o corpo discente e $50 \%$ do corpo docente não estarem informados sobre as ações do Pró-Saúde, pôde-se perceber que o Pró-Saúde influenciou de forma positiva o ensino odontológico.

\section{DESCRITORES}

Pró-Saúde. Cenários de Prática. Sistema Único de Saúde. Atenção Básica. $\mathbf{0}$ s limites da interpretação biomédica do adoecimento das pessoas acompanhou durante anos a formação profissional em Odontologia, até que as Diretrizes Curriculares Nacionais propostas para os referidos cursos, sinalizaram uma mudança de perfil do profissional a ser formado, incorporando novos aspectos em sua formação generalista, humanista, crítico e reflexiva. ${ }^{1}$

Ao esperar por essas mudanças propostas pelo governo, os dentistas recém-formados acabavam ficando como mão de obra passageira até que tivessem condições de sair e se especializar, e migrassem para níveis mais complexos. Então o Ministério da Saúde, em parceria com o Ministério da Educação, elaboraram o Programa Nacional de Reorientação Profissional em Saúde, a fim de estruturar as medidas propostas pelas Diretrizes Curriculares Nacionais, mudando os cenários de prática da graduação, aproximando-os da realidade dos futuros cirurgiões-dentistas e tentando atraí-los a ficar e se estabilizar.

A presente pesquisa centra seu olhar sobre a diversificação dos cenários de prática na formação em Odontologia e no impacto que as ações subsidiadas pelo Pró-saúde I e II promoveram nestes cenários de formação, enquanto política pública, e na sua contribuição para o processo de mudança pedagógica das instituições formadoras, sob o ponto de vista de gestores de unidades de ensino.

\section{REVISÃO DE LITERATURA}

A formação profissional em saúde deve englobar a importância dos diferentes níveis de complexidade, então é preciso que os cursos destaquem as importâncias das especialidades como um todo, na pretensão de se obter um profissional generalista.

Abraham et al. ${ }^{2}$ comparando as percepções dos 
alunos do curso de medicina em Melaka Manipal Medical College (MMMC) (Manipal Campus, Índia), percebeu que os alunos dos períodos mais avançados estavam desmotivados, e os alunos dos períodos iniciais, estavam mais empolgados, e incentivados, devido a variedade de cenários práticos presentes nesses.

Ferreira et al. ${ }^{3}$ afirmam que além da motivação, a mudança de cenários práticos ajuda na percepção do processo saúde-doença, quando os alunos são levados até as Unidades Básicas de Saúde (UBS), a fim de entender o funcionamento, e a realidade da população, formando assim profissionais mais críticos e reflexivos, seguindo assim o pensamento de Cabral et al., ${ }^{4}$ que afirmam que os estudantes e demais profissionais envolvidos nesse processo se tornam, não só profissionais de determinadas áreas, mas sim promotores de saúde, o que é o mais importante, que são profissionais generalistas, capazes de se adaptar ao ambiente, atender a demanda da população e ainda, controlar os níveis de saúde-doença, através da prevenção da doença e não só da cura da mesma, o que coincide com as afirmativas de Feuerwerker, Costa e Rangel. ${ }^{5}$

Durante a década de 90 , iniciou-se a mudança nas instituições de ensino superior, na tentativa de aproximá-las aos serviços públicos, porém a efetivação dessas alterações nas matrizes curriculares só aconteceu com a implantação das Diretrizes Curriculares Nacionais (DCNs) em 2002, como resultado da Lei de Diretrizes e Bases da Educação n 9394/96 e pela Reforma Sanitária Brasileira, que incentivou a interação ensino-serviço-comunidade. Essas diretrizes têm como objetivo encorajar o reconhecimento de habilidades, conferências e conhecimento obtido fora do ambiente escolar, fortalecendo a interação da teoria com a prática dos serviços e valorizando os estágios e participações em atividades da extensão. ${ }^{6}$

A inserção dos alunos nos cenários de prática, guiados pelos estágios curriculares, permite ao acadêmico aprender a valorizar o coletivo com um olhar mais reflexivo sobre os usuários, além de obter experiência em trabalho de equipe e elaborar ações de promoção de saúde ao invés de apenas tratar ou curar a doença. ${ }^{7}$

Alguns elementos que contextualizavam a diversificação dos cenários de aprendizagem acadêmica no Brasil, como as Diretrizes Curriculares Nacionais, para os cursos em graduação na área da saúde, o AprenderSUS, que engloba a produção de habilidades técnicas e o entendimento dos objetivos do SUS, o ProMed são objetos apontados por Carvalho ${ }^{8} \mathrm{e}$ posteriormente o Programa Nacional de Reorientação na Formação Profissional em Saúde (Pró-Saúde), como instrumentos de incentivo à formação, que tem como princípio direcionar o preceito constitucional que declara "a saúde como um direito e um dever do estado" garantindo atenção a saúde de forma universal, integral e com qualidade a todos os brasileiros. E outro objetivo do programa é formar equipes interdisciplinares, capazes gerir e organizar o sistema, respondendo aos desafios desta dinâmica complexa e pouco organizada presente nos processos de trabalho em saúde.

O Ministério da saúde, através da Secretaria de Gestão do Trabalho e da Educação em Saúde (SGTES), elaborou, em conjunto com a Secretaria de Educação Superior (SESu) e com o Instituto Nacional de Estudos e Pesquisas Educacionais Anísio Teixeira (INEP) do Ministério da Educação, o Programa Nacional de Reorientação da Formação Profissional em Saúde (Pró-Saúde), a fim de permitir a intervenção do governo nos cenários de prática das Instituições de Ensino Superior, contempladas com o programa, na tentativa de incluí-las no serviço público, aproximando os futuros profissionais da realidade do mercado e das demandas da população, e incentivando-os a fortalecer o Sistema Único de Saúde (SUS). A proposta levou em consideração a sustentação estrutural sugerida pelas Diretrizes Curriculares Nacionais, estabelecidas pelo Conselho Nacional de Educação para as profissões de saúde, bem como o Sistema Nacional de Avaliação da Educação Superior (SINAES). ${ }^{8}$

Através das Portarias Interministeriais MS/MEC $\mathrm{n}^{\circ} 2.101$, de 03 de novembro de 2005 e MS/MEC $\mathrm{n}^{\circ}$ 3.019 , de 27 de novembro de 2007, os cursos de graduação em odontologia, foram contemplados com o Programa Nacional de Reorientação da Formação Profissional em Saúde (Pró-Saúde), a fim de que os processos de reorientação acompanhassem simultaneamente distintos eixos, proporcionando uma escola formadora integrada ao serviço público de saúde, fortalecendo assim, a capacidade gestora e resolutiva do SUS. ${ }^{9}$

Preocupados em garantir um serviço público de qualidade, o governo federal, sintonizou as necessidades da população com a formação profissional, levando o Ministério da Educação em Parceria com o Ministério da Saúde, criarem formas de reorientar a formação profissional em saúde, reestruturando seus eixos de formação acadêmica, integrando o en- 
sino ao serviço público, assegurando assim uma abordagem geral do processo saúde-doença, enfatizando a atenção básica. Para isso, estipularam vários objetivos como: ${ }^{6}$

- reorientar o processo de formação dos profissionais da saúde, de modo a oferecer à sociedade profissionais habilitados para responder às necessidades da população brasileira e à operacionalização do SUS;

- estabelecer mecanismos de cooperação entre os gestores do SUS e as escolas, visando à melhoria da qualidade e à resolubilidade da atenção prestada ao cidadão, à integração da rede pública de serviços de saúde e à formação dos profissionais de saúde na graduação e na educação permanente;

- incorporar, no processo de formação da área da Saúde, a abordagem integral do processo saúde-doença, da promoção da saúde e dos sistemas de referência e contra-referência;

- ampliar a duração da prática educacional na rede pública de serviços básicos de saúde, inclusive com a integração de serviços clínicos da academia no contexto do SUS.

A fim de orientar as instituições durante as ações de reorientação profissional, foram elaborados três eixos para as ações desenvolvidas pelo Pró-Saúde, tendo cada um dos vetores específicos:

- Eixo A - Orientação Teórica:

A1 - Determinantes de saúde e doenças

A2 - Pesquisa ajustada a realidade local

A3 - Educação permanente

- Eixo B - Cenários de Prática:

B1 - Integração ensino-serviço

B2 - Utilização dos diversos níveis de atenção

B3 - Integração dos serviços próprios das IES com os serviços de saúde

- Eixo C - Orientação Pedagógica:

C1 - Integração básico-clínica

C2 - Análise crítica dos serviços

C3 - Aprendizagem ativa

Ao citar orientação teórica, a proposta do programa é trabalhar os determinantes de saúde, estudos clínico-epidemiológico e determinação biológico-social da doença, tudo sustentado por evidências que permitem avaliar criticamente o processo saúde-doença, além de redirecionar intervenções e protocolos. Objetiva-se também o estudo de questões administrativas do SUS, a fim de melhorar a capacidade gestora, e de tomada de decisões dos futuros profissionais. Para isso, o documento ministerial destaca os seguintes objetivos deste eixo: ${ }^{10}$

- priorizar os determinantes de saúde e os biológicos e sociais da doença;

- pesquisa clínica-epidemiológica baseada em evidências para uma avaliação crítica do processo de Atenção Básica;

- orientação sobre melhores práticas gerenciais que facilitem o relacionamento;

- atenção especial à educação permanente, não restrita à pós-graduação especializada.

Ao citar cenários de prática, objetiva-se desinstitucionalizar as atividades práticas realizadas pelas instituições de ensino, ou seja, fazer com que ocorram fora das universidades, em ambulatórios, domicílios, comunidade, unidades básicas de saúde, escolas e outros lugares possíveis, contradizendo a tendência de fazer com que as atividades práticas, ocorram apenas em clínicas, laboratórios e locais de alto custo de utilização. Os cenários de formação profissional devem ser diversificados, somando além de equipamentos de saúde, equipamentos educacionais e comunitários. Essa mudança, deve acontecer desde os períodos iniciais, a fim de que os acadêmicos possam assumir situações problemáticas de acordo com seu grau de autonomia. Este eixo, apresenta alguns objetivos como: ${ }^{10}$

- utilização de processos de aprendizado ativo (nos moldes da educação de adultos);

- aprender fazendo e com sentido crítico na análise da prática clínica;

- o eixo do aprendizado deve ser a própria atividade dos serviços;

- ênfase no aprendizado baseado na solução de problemas;

- avaliação formativa e somativa.

A orientação pedagógica refere-se a uma mudança na forma de ensino aprendizagem, propondo desafios que possam ser superados pelo estudante, possibilitando-os que sejam construtores de conhecimento, tendo assim o professor como orientador e facilitador deste processo. Um preceito pedagógico clássico é o "aprender-fazendo", e a proposta é justamente o contrário, "fazer-aprendendo" a fim de estimular o aluno a produzir conhecimento, gerando a dinâmica ação-reflexão-ação. Então o documento ministerial destaca os objetivos deste eixo: ${ }^{10}$ 
- diversificação, incluindo vários ambientes e níveis de atenção;

- maior ênfase no nível básico com possibilidade de referência e contra-referência;

- importância da excelência técnica e relevância social;

- ampla cobertura da patologia prevalente;

- interação com a comunidade e alunos, assumindo responsabilidade crescente mediante a evolução do aprendizado;

- importância do trabalho conjunto das equipes multiprofissionais.

Para uma universidade ser selecionada pelo Pró-Saúde, tem que obedecer a uma série de critérios, como tratamento equilibrado dos três eixos (orientação teórica, cenários de prática e orientação pedagógica), ter clareza na abordagem dos determinantes sociais de saúde/doença e no esquema curricular do curso, possibilidade de articulação com o serviço de saúde do município, integração com o hospital de ensino nas redes de serviço e indicação dos parâmetros de avaliação. Então, a partir destes requisitos, foram selecionados 89 cursos para o Pró-Saúde I, sendo 24 de odontologia, 27 de enfermagem e 38 de medicina, e para o Pró-Saúde II foram selecionados 265 cursos das demais áreas da saúde, impactando sobre aproximadamente 97.000 alunos de 14 áreas envolvidas. ${ }^{11}$

Escolas contempladas com o Pró-Saúde, têm em sua carga horária, um período destinado ao SUS e as visitas às Unidades Básicas de Saúde, sendo esta maior ou menor, de acordo com o nível de importância dado por seus representantes. E percebe-se que esta diversificação de cenários vem acontecendo de forma efetiva, melhorando assim a formação humanística dos novos profissionais que serão lançados no mercado de trabalho, tornando-os aptos a responder às demandas da população. ${ }^{12}$

Palmier et al..$^{13}$ relatam as experiências da disciplina de Ciências Sociais Aplicadas a Saúde, na Faculdade de Odontologia da Universidade Federal de Minas Gerais, respondendo às recomendações das Diretrizes Curriculares Nacionais e do Pró-Saúde, e durante esse período, percebeu-se que apenas uma pequena parte dos estudantes utiliza o Sistema Único de Saúde, e a maioria descartaria trabalhar no serviço público, devido as informações passadas pela mídia em relação a precariedade de materiais, baixos salários, e outros aspectos transmitidos pela mídia, que acabam gerando um preconceito, que se não for bem trabalhado, muitos profissionais vão continuar seguindo outras áreas e sem interesse no SUS. O Pró-Saúde serviu para motivar os futuros profissionais a se capacitarem e prestar serviço a saúde pública.

Silva ${ }^{14}$ afirma em pesquisa realizada em Valença, RJ, que parte da população, muitas vezes por não ter informação o suficiente, acaba condicionando a insatisfação com o serviço de prestação de saúde com a insuficiência do mesmo. Porém boa parte tem consciência de seus direitos, mas acreditam que a não prestação do serviço muitas vezes se deve ao fato de haver favorecimento de terceiros, ou por falta de recursos, o que leva a crer num possível despreparo dos profissionais em relação a formação ética e humanista, que é uma das principais linhas de defesa do Pró-Saúde e das DCNs.

\section{METODOLOGIA}

Trata-se de um estudo transversal, descritivo e exploratório, que utiliza a abordagem quantitativa para coleta, e a estatística descritiva para a análise e interpretação dos dados levantados.

$\mathrm{O}$ estudo trabalhou com um universo amostral formado por coordenadores e/ou responsáveis pedagógicos pelo Curso de Graduação em Odontologia (Coordenadores de Colegiado de Curso, Diretores de Faculdades ou representantes legais, dependendo da organização administrativa da instituição) contemplados com o Pró-Saúde I ou II, componente estabelecido como critério de inclusão no estudo.

Buscando minimizar perdas ao longo do processo, foi previamente feito contato virtual, telefônico e por correspondência oficial com as pessoas envolvidas como voluntárias na pesquisa, no sentido de incentivar sua participação.

Foi aplicado um questionário fechado virtual aos coordenadores dos cursos de Odontologia, no formato de survey, cujas respostas foram reenviadas automaticamente e alimentaram um banco de dados no domínio do Google docs ${ }^{\circledR}$.

O projeto de pesquisa foi encaminhado para análise ao Comitê de Ética em Pesquisa da Universidade Severino Sombra, como determina a resolução 196/96 do Conselho Nacional de Saúde para pesquisas com seres humanos, e aprovado sob parecer $\mathrm{n}^{\circ}$ 008/2012-1.

As informações somente passaram a ser coletadas após prévia autorização dos participantes da pesquisa, por meio do Termo do Consentimento Livre e Esclarecido online, devidamente aprovado 
pelo referido Comitê de Ética em Pesquisa, garantindo assim, o sigilo e o anonimato do depoimento dos participantes, visando a proteger sua privacidade e de suas instituições.

\section{RESULTADOS}

Apesar de haver 45 cursos de odontologia contemplados com o Pró-Saúde, apenas 13 responderam ao instrumento, justificado por alguns estarem de licença, greve em universidades públicas, entre outros motivos. Desses, 11 cursos foram contemplados com o Pró-Saúde I e 2 com o Pró-Saúde II.

Em 70\% dos cursos (Gráfico 1), o Pró-Saúde trouxe uma readequação da estrutura física da rede de serviços e a valorização dos cenários de prática

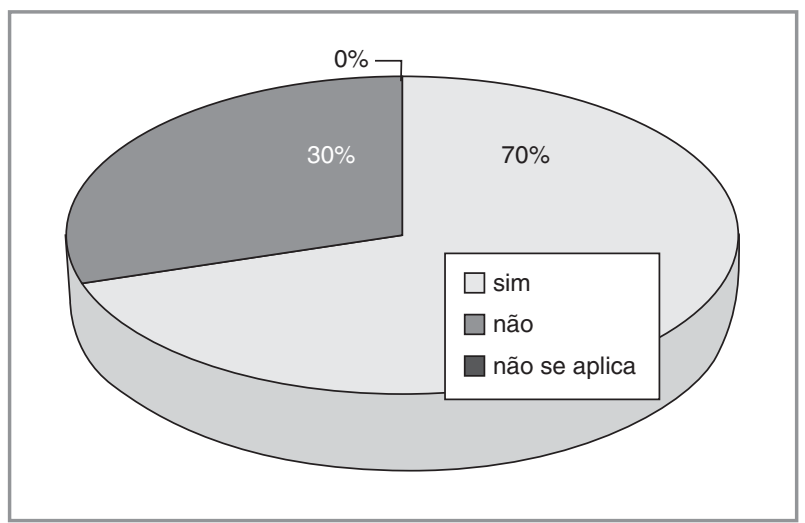

Gráfico 1 - O Pró-Saúde promoveu a integração ensino/ serviço envolvendo a comunidade como espaço social participativo?

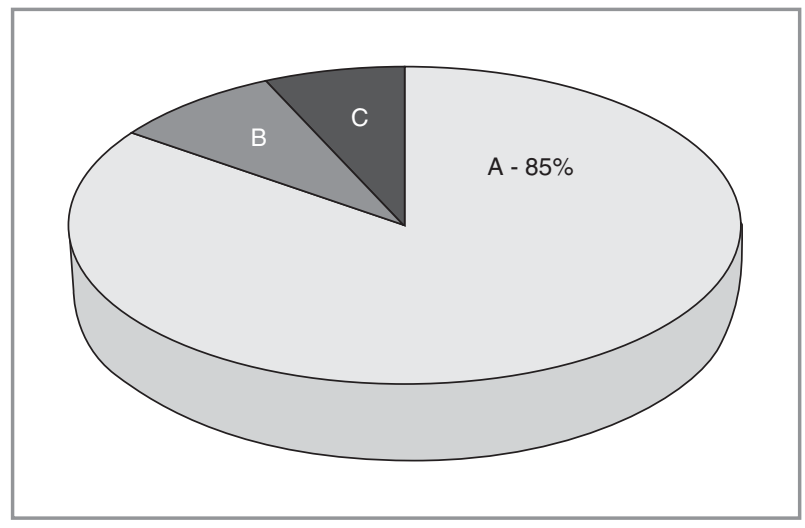

Gráfico 3 - O Pró-Saúde contribui com A: readequação da estrutura física da rede e valorização dos cenários de prática, mudança da matriz curricular e nos métodos de ensino/aprendizagem adotados pelos docentes, B: readequação da estrutura física da rede e valorização dos cenários de prática somente, C: mudança da matriz curricular somente. para a aprendizagem discente, com mudança da matriz curricular e dos métodos de ensino/aprendizagem adotados pelos docentes.

O Pró-Saúde promoveu em 85\% (Gráfico 2) dos cursos a integração ensino-serviço envolvendo a comunidade como o espaço social participativo. Em 85\% (Gráfico 3) dos cursos o Pró-Saúde promoveu a integração entre a orientação teórica do curso e prática nos serviços públicos.

Todos os coordenadores afirmaram que os alunos estão mais preparados para o enfrentamento dos desafios profissionais.

Em 70\% (Gráfico 6) dos cursos houve um incremento na carga horária total destinada aos estágios supervisionados na rede de serviço do SUS e em

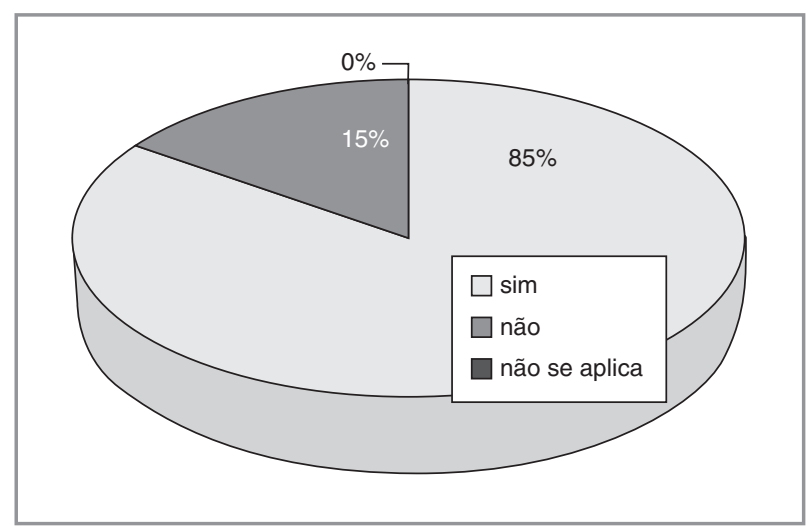

Gráfico 2 - O Pró-Saúde promoveu a integração entre a orientação teórica do curso e a prática nos serviços públicos de saúde com a participação de demais núcleos disciplinares?

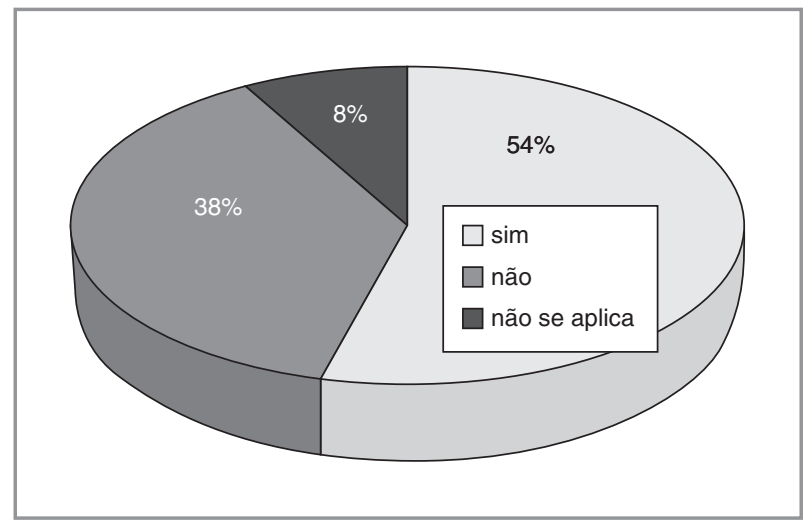

Gráfico 4 - O corpo docente do curso de Odontologia conhece as ações do Pró-Saúde na IES? 


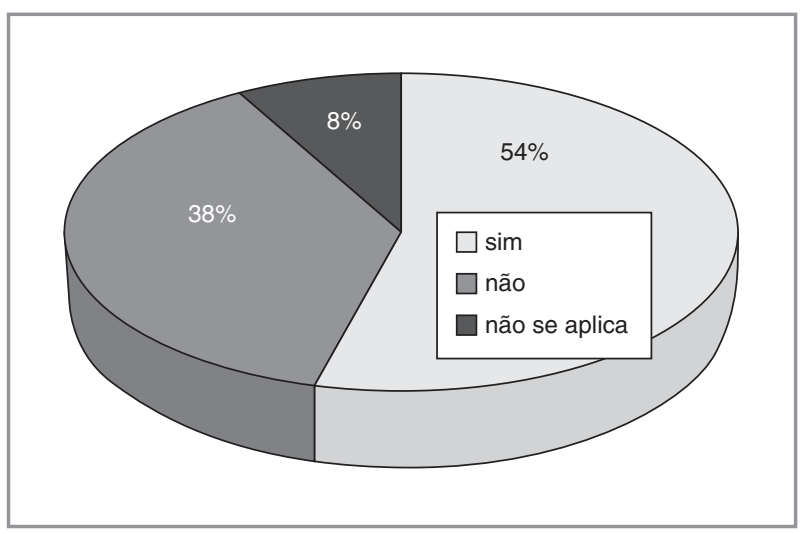

Gráfico 5 - Todo o corpo discente do curso de Odontologia conhece as ações do Pró-Saúde na IES?

54\% (Gráficos 4 e 5) dos cursos os corpos docente e discente conhece as ações do Pró-Saúde, em 38\% não conhecem e $8 \%$ não souberam responder.

Em todos os cursos o Pró-Saúde foi capaz de valorizar a atuação profissional na atenção primária à saúde no meio acadêmico.

\section{DISCUSSÃo}

Abraham et $a .^{2}$ afirmam que estudantes estão mais empolgados nos períodos iniciais do que nos finais, devido a uma provável variedade de cenários práticos e teóricos existentes no início do curso, concordando com Ferreira et al..$^{3}$ que acreditam na motivação dos estudantes, através da diversidade de cenários práticos.

Cabral et al. ${ }^{4}$, afirmam que os estudantes e demais profissionais envolvidos nesse processo se tornam, não só profissionais de determinadas áreas, mas sim promotores de saúde, o que é o mais importante, que são profissionais generalistas, capazes de se adaptar ao ambiente, atender a demanda da população e ainda por cima, controlar os níveis de saúde-doença, através da prevenção da doença e não só da cura da mesma, o que coincide com as afirmativas de Feuerwerker, Costa e Rangel. ${ }^{5}$ Além desses autores, Torres $^{1}$ afirma também que muitas vezes o profissional aborda o processo saúde-doença, baseado apenas nas consequências da doença, deixando passar seus determinantes sociais, fazendo com que a prevenção seja dificultada.

Morita e Krigger ${ }^{7}$ afirmam que profissionais formados em locais sem diversificação de cenários saem despreparados para trabalhar em equipe e com o conceito de apenas curar a doença, sem entendê-la e pensar na melhor forma de previnir, o que os torna desatuali-

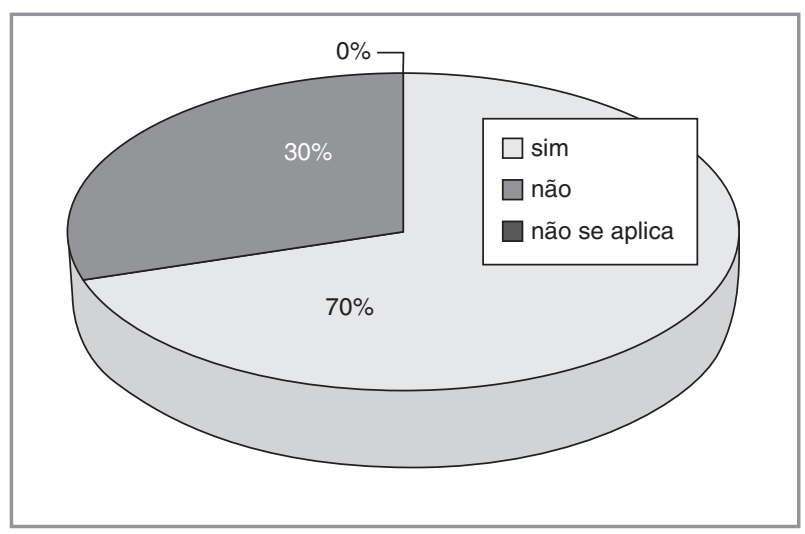

Gráfico 6 - Houve um incremento na carga horária total do curso destinada aos estágios supervisionados na rede de serviços do SUS com o incentivo do Pró-Saúde?

zados, e estes profissionais, que não conseguem lidar com os materiais que estão à disposição, ou seja, são treinados apenas para trabalhar com determinados materiais. Esse despreparo fica evidente ao chegar a uma UBS, pela insatisfação demonstrada pela população com o SUS, culpando o sistema por uma falha do profissional, o que motiva o governo a criar programas para a preparação dos profissionais em saúde, começando desde a sua formação, como é o caso do Pró-Saúde. ${ }^{14}$

Cabral $e t a l .^{4}$ relatam a dificuldade da triangulação ensino-serviço-comunidade, necessária para o entendimento das reais necessidades da população, enquanto Silva et $a l .{ }^{12}$, destacam que as escolas contempladas com o Pró-Saúde reservam um período para o entendimento do SUS, além de visitas domiciliares, e conhecimento das Unidades Básicas de Saúde, o que facilita a aplicação dessa triangulação proposta anteriormente.

O documento ministerial ${ }^{8}$ afirma a preocupação do Estado em proporcionar um serviço público de qualidade, por meio de uma parceria entre o Ministério da Saúde e o Ministério da Educação para elaborar programas que incentivassem e reorientassem a formação profissional em prol da saúde pública, como o PROMED e o Pró-Saúde que foram direcionados a fim de proporcionar e incentivar o ensino e o entendimento do SUS e das necessidades da população, defendendo o direito a saúde através de mudanças durante a graduação, para que os futuros profissionais tenham capacidade de melhorar cada vez mais o serviço de prestação a saúde, além de colaborar na organização da gestão e torná-la apta e estruturada para responder as demandas da população. 
A formação ética é fundamental para a experiência profissional, pois quando relacionada a capacidade autônoma e a reflexão crítica, o profissional passa a tomar decisões coerentes e enxerga o paciente como um todo, conforme Palmier et al. ${ }^{13}$ ao relatar que o tradicionalismo na educação gera uma desorganização no processo de ensino-aprendizagem, levando a uma "produção de conhecimento" altamente desestimulante para o acadêmico, conflitando com um dos principais objetivos do Pró-Saúde que é o estímulo a busca pelo conhecimento e pelo preparo em todas as áreas, tornando-se um profissional generalista e apto a promover a saúde, seja através de palestras, diálogos e outras formas.

O documento ministerial ${ }^{9}$ afirma que muitas vezes a rotatividade de profissionais nas Unidades Básicas de Saúde, se deve a falta de preparo profissional que muitas vezes não atinge às demandas da população de forma adequada, o que levou o Ministério da Saúde, em parceria com o Ministério da Educação a perceberem que seria necessário reorientar a formação profissional em saúde pública, e para auxiliar na organização dessas práticas nas Instituições de Ensino Superior foram criadas as Diretrizes Curriculares Nacionais que conforme o $\mathrm{Brasil}^{6}$ propunham uma flexibilidade na organização dos currículos além de promover a interação ensino-serviço-comunidade.

\section{CONCLUSÃO}

Com a presente pesquisa, apesar de um estudo preliminar, pôde-se já perceber que o Pró-Saúde está sendo eficaz e eficiente para os cursos de Odontologia, pois segundo os coordenadores participantes, os cursos vêm modificando a matriz curricular, acrescentando uma carga horária extra, dedicada ao conhecimento das ações do SUS, e das Unidades Básicas de Saúde.

Os cenários de prática sofreram e ainda sofrem alterações, sendo assim os alunos tem vivenciado melhor a realidade do SUS, e aos poucos apagando ou pelo menos melhorando a imagem negativa transmitida pela mídia, apesar de ainda existirem muitas mudanças a serem feitas.

Um aspecto negativo da pesquisa é o fato de apesar das presentes melhoras trazidas pelo Pró-Saúde, grande parte dos professores e alunos dos cursos contemplados, não conhecem as ações do Pró-Saúde, apesar de vivenciá-las, não dando assim o devido reconhecimento que o programa merece.

O Pró-Saúde vem melhorando a percepção destes novos profissionais, que estão sendo lançados no mercado de trabalho melhor preparados para atender às demandas da população, tendo um olhar mais crítico, reflexivo e humanístico, capazes de enxergar o paciente como um todo, realizar ações de promoção de saúde e prevenção de doenças, com um melhor entendimento do processo saúde-doença, além de serem mais generalistas, atendendo de fato as demandas da população.

\section{ABSTRACT}

The impact of practice scenarios proposed by ProHealth in dentistry training

The Health Education and Work Management Department (SGTES) under the Ministry of Health drafted the National Program of Professional Health Training Realignment (Pro-Health) as an incentive for healthcare courses to promote a change in teaching practice scenarios, for the purpose of introducing students into the Health System, and, in turn, showing them the real demands of the population and preparing them for the job market. The aim of this study was to analyze the dentistry courses in Brazil where the Pro-Health program was implemented. Course coordinators were interviewed on the impact of the teaching program and on how well-informed they were about the actions of Pro-Health. A structured questionnaire was applied online to 45 coordinators of undergraduate dentistry courses in Brazil, following approval by the USS Ethics and Research Committee (protocol number 008/2012-1). Preliminary results showed that $25 \%$ perceived that the program influenced both the physical structure and the core curriculum, in that an extra course load was added in $67 \%$ of the universities, to cover internships in Basic Health Units. Although the coordinators claimed that the student body and $50 \%$ of the faculty in $50 \%$ of universities were not informed about the actions of Pro-Health, it could be seen that Pro-Health has indeed influenced dental education positively.

\section{DESCRIPTORS}

Pro-Health. Practice Scenarios. Unified Health System. Primary Care. •

\section{REFERÊNCIAS}

1. Torres, MCMB. Interdisciplinaridade Contemplada no Projeto Pedagógico no Ensino da Odontologia: uma necessidade. IN: Rangel, JNM (org.) Caminhos Interdisciplinares na Odontologia. Rio de Janeiro: Editora Rubio, 2006, 72 p.

2. Abranam, R; Ramnarayan, K; Vinod, P; Torke, S. Students' 
perceptions of learning environment in an Indian medical school. BMC Med Educ; 2008. p. 8-20.

3. Ferreira, RC; Silva, RF; Aguera, CB. Formação do Profissional Médico: a Aprendizagem na Atenção Básica de Saúde. Revista Brasileira de Educação Médica. 2007. n. 52 v.1, supl. 31, p. 52 -59 .

4. Cabral, PE; Machado, JLM; Machado, VMP; Pompílio, MA; Vinha, JM; Ayash, W; Mattos, MCI. Serviço e Comunidade, Vetores para a Formação em Saúde: o Curso de Medicina da Uniderp. Rev. bras. educ. méd; 2008. n. 375, v.3, supl. 32, p. $374-382$;

4. Feuerwerker L. Educação dos profissionais de saúde hoje: problemas, desafios perspectivas e as propostas do Ministério da Saúde. Rev ABENO. 2003;3:24-7.

5. _ Ministério da Educação. Diretrizes Curriculares Nacionais dos Cursos de Graduação em Farmácia e Odontologia. CNE/CES no 2, de 19 de fevereiro de 2002. Disponível em: http://portal.mec.gov.br/cne/arquivos/pdf/CES1300. pdf Acesso em 10/02/10.

6. Morita, MC; Kriger, L. Mudanças nos cursos de odontologia e a interação com o SUS. Revista da ABENO; 2004. n. 4, v.1, p. $17-21$.

7. .Ministério da Saúde. Ministério da Educação. Programa Nacional de Reorientação da Formação Profissional em Saúde - Pró-Saúde: objetivos, implementação e desenvolvimento potencial/Ministério da Saúde, Ministério da Educação. Brasília: Ministério da Saúde, 2007. 86 p.: il. - (Série C Projetos, Programas e Relatórios).

8. _ Ministério da Saúde. Ministério da Educação. Porta- ria Interministerial $\mathrm{n}^{\circ} 2.101 \mathrm{DE} 3$ de novembro de 2005. Disponivel em: http://www.abem-educmed.org.br/publicacoes/ boletim_virtual/volume_10/portaria_pro_saude.pdf Acesso em: 09/12/09.

9. BRASIL. Ministério da Saúde. Secretaria de Gestão do Trabalho e da Educação na Saúde. Programa Nacional de Reorientação da Formação Profissional em Saúde (Pró - Saúde), 2008. Disponível em: http://www.prosaude.org/publicacoes/index.php Acesso em: 09/12/09.

10. BRASIL. Ministério da Saúde. Secretaria de Gestão do Trabalho e da Educação na Saúde. Programa Nacional de Reorientação da Formação Profissional em Saúde (Pró - Saúde), 2009. Disponível em: http://www.prosaude.org/not/prosaude_maio2009/prosaude.pdf Acesso em: 21/06/12.

11. Silva MAM, Amaral JHL, Senna MIB, Ferreira EF. O Pró-Saúde e o incentivo à inclusão de espaços diferenciados de aprendizagem nos Cursos de Odontologia no Brasil. Revista Interface, Comunicação, Saúde e Educação (UNIFESP) 2012. v.16, n.42.

12. Palmier AC, Amaral JHL, Werneck MAF, Senna MIB, Lucas SD. Inserção do aluno de odontologia no SUS: contribuições do Pró-Saúde. Rev. bras. educ. méd. 2012. n .36, supl.2, p. 152157.

13. Silva MAM, Ferreira EF, Silva GA. O direito à saúde: representações de usuários de uma unidade básica de saúde. Physis [online]. 2010. n.4, vol.20, ISSN 0103-7331.

Recebido em 08/10/2012 Aceito em 10/12/2012 